\title{
Enhancement of conditioned inhibition via an extinction treatment
}

\author{
PAUL L. DEVITO \\ Saint Joseph's University, Philadelphia, Pennsylvania \\ and \\ HARRY FOWLER \\ University of Pittsburgh, Pittsburgh, Pennsylvania
}

\begin{abstract}
Rats were used in a conditioned-suppression paradigm to determine whether an extinction treatment would enhance a moderately developed conditioned inhibitor (CS-). To dissipate unconditioned suppression to the training stimuli, the subjects were first habituated to the stimuli and then given Pavlovian conditioned-inhibition (CI) training involving reinforced presentations of a clicker and nonreinforced compound presentations of that stimulus and the intended CS-, either a light or a tone. Thereafter, experimental subjects received presentations of their CS - by itself, whereas controls received no further training. Following the occurrence and loss of conditioned suppression to the CS - in the extinction phase, summation and retardation tests showed enhanced CI for the experimental subjects relative to both the controls and their own earlier levels of inhibitory performance. In fact, the enhanced inhibition for the experimental subjects approximated that shown by a comparison group for which the CS - had been strongly developed as an inhibitor. These findings suggest that an excitatory representation is associated with the CSearly in CI training, and that subsequent presentations of the CS- by itself strengthen its inhibitory effect by allowing it to be nonreinforced in the presence of that representation.
\end{abstract}

A consistent finding that has plagued the otherwise robust Rescorla-Wagner (1972; Wagner \& Rescorla, 1972) model of conditioning virtually from its inception is the fact that conditioned inhibition (CI), unlike conditioned excitation (CE), cannot be extingiuished by the "standard" operation of presenting the conditioned stimulus (CS) by itself (e.g., DeVito \& Fowler, 1986; Lysle \& Fowler, 1985; Owren \& Kaplan, 1981; Pearce, Nicholas, \& Dickinson, 1982; Rescorla, 1982; Witcher \& Ayres, 1984; Zimmer-Hart \& Rescorla, 1974). In line with that observation and the opposite operations that are used to establish CI and CE, we questioned the appropriateness of using a CS-alone procedure to extinguish CI (DeVito \& Fowler, 1986). This procedure is effective in extinguishing $\mathrm{CE}$ primarily because it removes the contingent reinforcer, that is, the unconditioned stimulus (US), that is used to develop an excitatory CS (CS+). However, when the CS-alone procedure is used following CI training, it does not remove the contingent nonreinforcer, that is, the absence of the US, that is used to develop and inhibitory CS (CS-). Instead, CS-alone

The research was supported in part by a Saint Joseph's University Faculty Research grant to Paul $\mathbf{L}$. DeVito and by a National Instinutes of Health grant (MH24115) and University of Pittsburgh Research Development Award to Harry Fowler. Correspondence concerning this article should be addressed to Paul L. DeVito, Department of Psychology, Saint Joseph's University, 5600 City Avenue, Philadelphia, PA 19131. training maintains the consequence of nonreinforcement for the CS - and can therefore maintain CI, or even strengthen it, as some investigators have noted (DeVito \& Fowler, 1986; Holland, 1985; Rescorla, 1982; Williams, Travis, \& Overmier, 1986).

The enhancement of CI produced by CS-alone training, however, is far from a ubiquitous effect; it has been limited to specific training and test arrangements and therefore does not necessarily reflect a strengthening of CI per se. For example, Rescorla (1982) found that following Pavlovian inhibitory $(\mathrm{A}+/ \mathrm{AB}-)$ training for pigeons in a keypecking task, presentations of the $B$ inhibitor by itself enhanced CI when B was subsequently tested with a new excitor, $C$, but not when $B$ was tested with the original excitor, A. Because a follow-up experiment showed that extinction of the $A$ excitor after A + /AB - training also enhanced B's inhibitory effect with $C$, Rescorla concluded that the enhancement stemming from B-alone presentations was due to B's disassociation from $\mathrm{A}$. By his argument, $\mathrm{AB}$ - compound training enabled $B$ to evoke a representation of $A$ (cf. Cunningham, 1981; Rescorla, 1981) that could attenuate B's CI effect with a new excitor, because the evoked representation of $\mathbf{A}$ would summate with $\mathbf{C}$ to generate greater excitation and thus less net inhibition. However, that effect would be eliminated if $A$ and $B$ were disassociated by the presentation of either $A$ alone or $B$ alone. Furthermore, there should be no effect of B-alone presen- 
tations on B's inhibitory effect with A since any evoked representation of $A$ would not summate with the excitatory effect produced by the A stimulus itself. Similar results and conclusions have recently been reported for the rat (Williams et al., 1986).

Although the above effects and interpretation are convincing, they do not preclude the possibility that CI can itself be strengthened by presenting $B$ alone. Indeed, by Rescorla's (1982) own argument, such presentations should allow B to be nonreinforced in the presence of A's excitatory representation, which is akin to B's nonreinforcement when trained in compound with the actual A stimulus. By this view, the absence of an enhancing effect of B-alone presentations on B's inhibitory effect with A merely reflects the fact that Rescorla and other investigators used a strongly, if not maximally, developed inhibitor on the presumption that B-alone presentations might extinguish CI. Consistent with our interpretation, two recent studies (DeVito \& Fowler, 1986; Holland, 1985 ) have indicated that when $B$ is only moderately developed as a CS - , and therefore does not completely inhibit A's excitatory effect, presentations of B by itself strengthen its inhibitory effect on A. However, the results of these more recent studies are open to question.

The enhancing effect reported by DeVito and Fowler (1986) was an ancillary finding on the pre- and post-Balone performance of a group that served as a control for others; there was no control for the group that received the same training and testing without $\mathrm{B}$-alone presentations. Holland (1985), on the other hand, used both a Balone group and a control group that received the same training and testing without $\mathrm{B}$-alone presentations. This comparison would seem satisfactory, but there is mounting evidence that mere exposure to a training context appreciably reduces CI to B (Henderson, 1978; Lysle \& Fowler, 1985), in contast to the neutral effect of no exposure at all (Lysle \& Fowler, 1985; Thomas, 1979). Further complicating both investigations is the fact that they established a moderately strong B inhibitor by using a light $\mathrm{CS}$ - for rats in a conditioned-suppression paradigm. For nocturnal beasts so trained, that type of stimulus is noted for producing pronounced unconditioned suppression and relatively weak CI (e.g., Kleiman \& Fowler, 1984; Lysle, 1983). Thus, it may have been that presentations of the light $C S$ - by itself merely reduced unconditioned suppression to $B$, resulting in less suppression to the $A B$ compound in test.

In view of the potential import of CS-alone training in implicating a reinforcement mechanism for $\mathrm{CI}$, we sought to replicate the enhancing effect of such training under conditions that were properly controlled. Rats in a conditioned-suppression paradigm were first given habituation trials to the intended CSs so as to attenuate unconditioned suppression to the stimuli. They then received excitatory conditioning to a clicker (stimulus A) followed by $\mathbf{A}+/ \mathbf{A B}-$ training involving a light $\mathbf{C S}-$ (stimulus B) for one pair of groups and a tone CS - for another pair. To compensate for differential rates of inhibitory conditioning to the B stimuli, the different pairs of groups received extended inhibitory conditioning to the light and limited inhibitory conditioning to the tone, with the result that both stimuli comparably but only moderately developed as inhibitory CSs. Thereafter, one group of each pair was given nonreinforced presentations of its respective CS -, whereas the other group of each pair was detained in holding cages and not exposed to the $\mathrm{CS}-$ or the training context. Like the B-alone tone group, a fifth group was also given inhibitory conditioning and extinction to the tone; however, that group received the same amount of CI training as the light groups and therefore had a strongly developed tone $\mathrm{CS}-$. This fifth group enabled us to determine the extent to which any enhanced CI from B-alone training for the moderately developed tone group approached an asymptote of $\mathrm{CI}$. Both summation and retardation tests were used to evaluate CI.

\section{METHOD}

\section{Subjects}

Forty experimentally naive male Sprague-Dawley rats, 90 days old and 350-400 $\mathrm{g}$ in weight, were purchased from the Holtzman Company. The subjects were individually caged in a colony room where the temperature was maintained at $22^{\circ} \mathrm{C}$ and the day-night cycle (12-h phase) was reversed through artificial illumination.

\section{Apparatus}

Four rodent chambers (Scientific Prototype Model A105), each measuring $25 \times 25 \times 25 \mathrm{~cm}$, were used for training and testing. Each chamber was housed in a sound- and light-attenuating cubicle $(50 \times 60 \times 90 \mathrm{~cm})$ located in a room adjacent to the programming equipment. A 100-W, 120-V bulb positioned behind a frosted glass plate in the ceiling of each cubicle was operated at $85 \mathrm{~V}$ ac to provide diffuse illumination of the chamber. An ambient sound level of $72 \mathrm{~dB}\left(\mathrm{re} .0002 \mathrm{dyn} / \mathrm{cm}^{2}\right)$ was effected within each chamber by operation of the cubicle's exhaust fan at $52 \mathrm{~V}$ ac.

Each chamber had clear Plexiglas side walls, sheet-metal top and end walls, and a grid floor consisting of $0.24-\mathrm{cm}$ bars spaced $0.75 \mathrm{~cm}$ apart. A 50-ml drinking tube (BRS/LVE Model DT-001), accessible through a $1-\mathrm{cm}$ oblong opening in the end wall of each chamber, was connected to a contact relay (BRS/LVE Model DR901/221-05) to allow the recording of licking responses. A 6-W, $120-\mathrm{V}$ bulb on the ceiling of each chamber was connected to two interval timers (Hunter Model 111-B) to provide a 20-sec visual CS: a light flashing equally on and off at the rate of $0.4 \mathrm{sec} /$ cycle. An 8.5- $\mathrm{cm}, 3.5-\Omega$ speaker (Quam Model 3A05) mounted on the drinking-tube wall was connected to an audio generator (BRS/LVE Model AU-902) to provide two 20-sec, 80-dB auditory CSs: a 1500$\mathrm{Hz}$ tone and a clicker pulsating at the rate of 8 clicks $/ \mathrm{sec}$. The grid floor of each chamber was connected to the output of a shock generator and scrambler (BRS/LVE Models SG 903 and SC 902) to provide an aversive US: a 0.5 -sec $1.3-\mathrm{mA}$ shock.

\section{Procedure}

Upon arrival, the subjects were given free access to food (Wayne Lab Blox) and water for 5 days. Thereafter, access was restricted to $8 \mathrm{~min} /$ day, with food available ad lib. Five days later, the subjects started the following training and test phases, throughout which water was available only during the daily 8 -min session in the experimental chamber.

Baseline and habituation training. All subjects were given 5 days of baseline training to establish steady licking of the drinking tube. Then they received 4 days of habituation training to the intended light, tone, and clicker CSs. The 20-sec CSs were each 
presented once per day, on a 2-min variable-time (VT) schedule, with their order counterbalanced across days to the extent possible. Licks were recorded during the 20 -sec period prior to each CS, during the 20-sec CS, and throughout the entire session. Stimulus presentations always occurred within the first 6.5-7 min of the 8-min session, while the subjects exhibited steady rates of licking. In addition, to avoid possible low pre-CS rates due to occasional pauses in drinking, each trial was monitored and not started until the subject was licking. Trial initiation and data collection were the same in all other phases. For a more detailed description of this procedure, see DeVito and Fowler (1986).

Excitatory and inhibitory conditioning. Excitatory conditioning to the 20-sec A stimulus (clicker) began following habituation training. Stimulus A always terminated with onset of the shock US. There were seven reinforced $(A+)$ trials, one the first day at $2 \mathrm{~min}$, and two per day thereafter at 1-3 and 4-6 min into the session. Following excitatory conditioning to $\mathrm{A}$, the subjects were randomly assigned to five groups $(n=8)$ for inhibitory conditioning to the 20 -sec B stimulus. For two groups, B was the light; for the other three groups, $B$ was the tone. On each day of inhibitory conditioning, all groups received two A + presentations and six nonreinforced presentations of $A$ in simultaneous compound with $B(A B-)$. Daily presentations of $A+$ and $A B$ - occurred randomly, on a 50-sec VT schedule, with the restriction that the serial position of $A+$ be balanced over consecutive four-trial blocks.

Inhibitory conditioning lasted 24 days for the two light-trained groups and for one of the three tone-trained groups. Because of differential rates of inhibitory conditioning to the light and tone, that amount of training resulted in a moderately developed CSfor the two light groups (designated ML) and a strongly developed CS - for the tone group (designated ST). Inhibitory conditioning was restricted to 7 days for the other two tone-trained groups (designated MT) so that the strength of their CS - approximated that for the ML groups. Inhibitory-conditioning days for the MT groups were randomly interspersed among those for the other three groups, with the restriction that the first and last days of inhibitory conditioning coincide for all groups. (On nonconditioning days, MT subjects received $8 \mathrm{~min}$ access to water in their home cages.)

Inhibitory extinction. Table 1 summarizes the treatments that the groups received in both the conditioning and extinction phases. As noted for the latter phase, one group of each pair of ML and MT groups was given nonreinforced presentations of its respective inhibitor by itself (Groups ML/E and MT/E); the other group of each pair received no exposure to either its inhibitory CS or the conditioning chamber during this phase (Groups ML/N and MT/N). The latter two groups were merely transported to the experimental room and detained in individual holding cages, identical to their home cages, where they were permitted $8 \mathrm{~min}$ access to water. Like the $M L / E$ and $M T / E$ groups, the strongly developed tone group also received nonreinforced presentations of its B inhibitor (Group

Table 1

Design of the Experiment

\begin{tabular}{lcccc}
\hline Group & Conditioning & Extinction & Test 1 & Test 2 \\
\hline ML/E & C+; C+, CL & L & C, CL & L +.5 \\
ML/N & C+; C+, CL & NTG & C, CL & L +.5 \\
MT/E & C+; C+, CT & T & C, CT & T+.5 \\
MT/N & C+; C+, CT & NTG & C, CT & T +.5 \\
ST/E & C+; C+, CT & T & C, CT & T +.5 \\
\hline
\end{tabular}

Note $-\mathrm{ML}=$ moderately developed light inhibitor; $\mathrm{MT}=$ moderately developed tone inhibitor; ST = strongly developed tone inhibitor. The symbols $E$ and $N$ designate groups that received extinction training $(E)$ or not (N). Stimuli $C, L$, and $T$ refer to a clicker, light, and tone conditioned stimulus, respectively; $+=100 \%$ reinforcement; $+.5=50 \%$ reinforcement; NTG $=$ no training or exposure.
$\mathrm{ST} / \mathrm{E})$. The three extinction-trained groups received five presentations of B per day, on a 50-sec VT schedule, for 6 consecutive days.

Summation and retardation testing. On each of the 2 days following the extinction phase, all groups were given two nonreinforced presentations of $A$ on a 4-min VT schedule. These sessions served to attenuate complete suppression to $A$. Thereafter, all groups received 1 day of summation testing during which there were two nonreinforced presentations each of $A$ and the $A B$ compound. Presentation of the four daily events occurred on a 2-min VT schedule in counterbalanced order: $\mathrm{A}, \mathrm{AB}, \mathrm{AB}, \mathrm{A}$.

On the following day, all subjects were given four nonreinforced presentations of their respective B inhibitor on a 2 -min VT schedule, thus providing a baseline for the measurement of conditioned suppression to $B$ over the next 8 days, when all groups were given an excitatory-conditioning retardation test. On each test day, B was presented twice, on a 4-min VT schedule, but was reinforced only on a random, $50 \%$ basis. That was intended to slow the rate of acquisition and reveal any differences among the groups. The specific stimulus events for the groups during both the summation and retardation tests are shown in the last two columns of Table 1.

\section{Statistical Treatment}

The data were typically subjected to a one-way analysis of variance for five groups. When the overall effect was significant $(\alpha=.05)$, preplanned orthogonal contrasts were used to form a $2 \times 2+1$ factorial that (1) compared the ML/E and MT/E groups with their no-extinction controls, $\mathrm{ML} / \mathrm{N}$ and $\mathrm{MT} / \mathrm{N}$, (2) compared the pair of ML groups with the pair of MT groups, (3) assessed the interaction of those extinction and stimulus factors, and (4) compared the moderately developed CS - groups with the strongly developed CS - group. In summation and retardation testing, an ancillary contrast based on the overall error term evaluated differences specific to the MT/E and ST/E groups.

\section{RESULTS}

\section{Baseline and Habituation Training}

Analyses of mean daily licks over the initial 5 days of baseline training and over the following 4 days of habituation training showed no reliable differences among the groups. Likewise, there were no reliable group differences in analyses of unconditioned suppression to the light, tone, and clicker CSs during habituation training. (Suppression scores were based on the ratio CS/[CS + preCS], where CS refers to the number of licks during the 20 -sec CS and preCS refers to the number of licks during the 20sec period prior.) However, the Day 1 habituation data showed a reliable stimulus effect $[F(2,70)=50.40$, $p<.001$ ]; the overall mean suppression ratios for the light, the tone, and the clicker were $.11, .09$, and .31 , respectively. Contrasts showed that the clicker produced reliably less suppression than the light and the tone $[F(1,70)=100.24, p<.001]$; however, the latter two were not different. By the last day of habituation training, unconditioned suppression to the stimuli had largely dissipated, but there still was a reliable difference among them $[F(2,70)=15.66, p<.001]$; the means for the light, the tone, and the clicker were $.34, .48$, and .43 , respectively. In this instance, the light produced greater suppression than the tone or the clicker $[F(1,70)=27.31$, $p<.001]$, with the latter two not being different. Im- 
portantly, there was no reliable suppression to the tone relative to the expected maximum ratio of .50 .

\section{Conditioning and Extinction Training}

All subjects developed suppression to A over the 4 days of excitatory conditioning; by the last day, the overall mean suppression ratio was .11. Analyses of suppression throughout this phase showed no reliable group effects.

The left panel of Figure 1 depicts the results of inhibitory conditioning. The data are presented as group mean differences in the suppression ratios for $A$ and $A B$ (i.e., $A B-A)$; hence, larger difference scores reflect a greater inhibitory effect of B. As shown, the ST group developed a high level of inhibition to $B$, whereas the MT and ML groups developed only moderate or modest levels of inhibition to B. Analysis of the data for the last day of inhibitory conditioning indicated reliable differences among the groups $[F(4,35)=10.11, p<.001]$. This outcome was primarily due to the difference between the ST group and the others $[F(1,35)=38.35, p<.001]$, as the difference between the ML and MT groups failed to reach significance. Likewise, there was no reliable difference between their prospective $\mathrm{E}$ and $\mathrm{N}$ subgroups and no interaction of that factor with the stimulus factor. Exactly the same effects were apparent in an analysis of suppression to the $\mathrm{AB}$ compound. In contrast, there were no reliable group differences in suppression to $A$ itself; by the last inhibitory conditioning day, the overall mean suppression ratio for $\mathrm{A}$ was .05 .

The right panel of Figure 1 shows group mean suppression to $B$ during the subsequent extinction phase. As indicated, there was little, if any, suppression to B for the $\mathrm{ST} / \mathrm{E}$ group throughout this phase. In contrast, the MT/E and $M L / E$ groups exhibited moderate amounts of suppression that decreased over the course of the B-alone trials. Hence, a repeated measures analysis of suppression to $B$ showed a reliable group effect $[F(2,21)=24.18$, $p<.001]$, a reliable trial effect $[F(2,42)=15.88$,
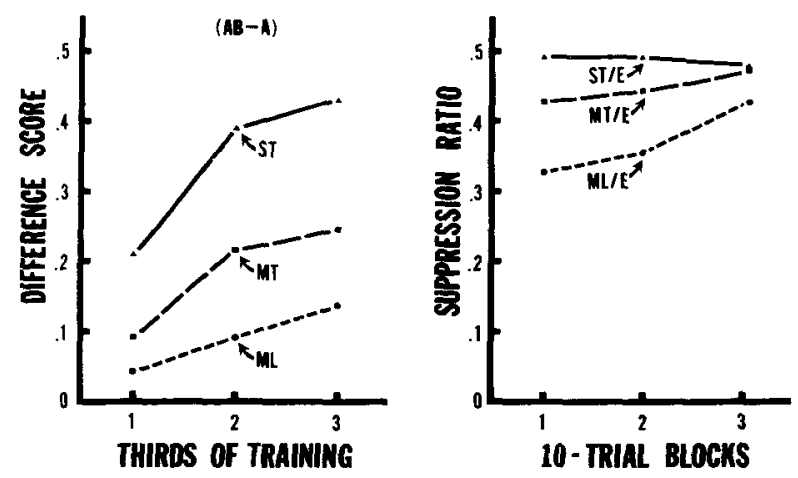

Figure 1. Mean difference in suppression to $A B$ and $A(A B-A)$ for the ST, MT, and ML groups during inhibitory conditioning (left panel), and mean suppression to the respective $B$ inhibitor for the ST/E, MT/E, and ML/E groups during extinction training (right panel). ST = strongly developed tone inhibitor: $M T=$ moderately developed tone inhibitor; $\mathrm{ML}=$ moderately developed light inhibitor; $\mathbf{E}$ designates groups that received extinction training.

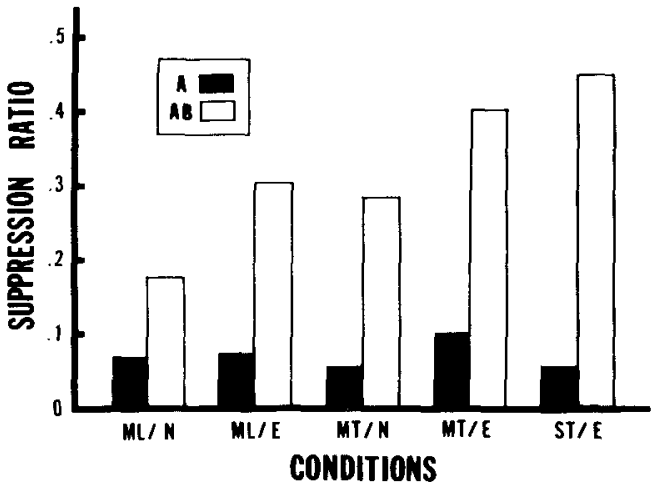

Figure 2. Mean suppression in the summation test to the original $A$ excitor and the respective $A B$ compound for each group. $M L=$ moderately developed light inhibitor; MT = moderately developed tone inhibitor; $S T=$ strongly developed tone inhibitor; $E$ and $N$ designate groups that received extinction training $(E)$ or not $(N)$.

$p<.001]$, and a reliable group $\times$ trial interaction $[F(4,42)=8.72, p<.001]$. Contrasts indicated that there was greater suppression to $B$ for the $\mathrm{ML} / \mathrm{E}$ group than for the MT/E group $[F(1,21)=23.91, p<.001]$ and greater suppression for those two groups than for the ST/E group $[F(1,21)=24.45, p<.001]$. Furthermore, although the group $\times$ trial interaction was marginally reliable for the MT/E and ML/E groups [linear $F(1,42)=$ $5.56, p<.05$ ], it was pronounced for those two groups as compared with that for the ST/E group [linear $F(1,42)$ $=28.97, p<.001]$. Thus, separate group assessments showed a reliable decrease in suppression to B for both the $\mathrm{ML} / \mathrm{E}$ group and the $\mathrm{MT} / \mathrm{E}$ group $\left[F_{\mathrm{S}}(1,42)>13.33\right.$, $p$ s $<.001]$ but no reliable effect for the ST/E group.

\section{Summation Testing}

There were no reliable group differences in suppression to $\mathrm{A}$ on the 2 days following the extinction phase when $A$ was presented by itself. On the last day, the overall mean suppression ratio to $A$ was .07 .

Figure 2 shows the results of the summation test involving nonreinforced presentations of both $A$ and $A B$. As indicated and confirmed by analysis, there were no reliable differences among the groups in suppression to $\mathrm{A}$. Group differences in suppression to $\mathrm{AB}$, however, were highly reliable $[F(4,35)=12.63, p<.001]$. Contrasts showed that suppression to $\mathrm{AB}$ was significantly less for the ML/E and MT/E groups than for their ML/N and MT/N controls $[F(1,35)=17.62, p<.001]$ and less for the pair of MT groups than for the pair of ML groups $[F(1,35)=10.46, p<.005]$. Those extinction and stimulus effects did not interact. In addition, the ST/E group showed less suppression to AB than did the four moderately trained groups $[F(1,35)=22.43, p<.001]$, but the more interesting and meaningful comparison, that between the ST/E and MT/E groups, showed no reliable difference. Thus, B-alone presentations elevated B's inhibitory effect for the MT/E group virtually to the level exhibited by the ST/E group. That enhancement was ap- 
parently not complete, though, because an analysis of difference scores (AB - A) indicated a reliable difference between the $\mathrm{MT} / \mathrm{E}$ and ST/E groups $[F(1,35)=$ 8.66, $p<.01]$. All other effects with the difference-score measure were the same as those for the $\mathrm{AB}$ measure.

To determine whether the summation-test results reflected a change in B's inhibitory effect for just the $\mathrm{ML} / \mathrm{E}$ and $\mathrm{MT} / \mathrm{E}$ groups, we compared group mean difference scores on the summation-test day with those for the last day of inhibitory conditioning (cf. Figure 1, left panel). That repeated measures analysis showed that although the day effect was unreliable overall, the group $X$ day interaction was significant $[F(4,35)=3.83$, $p<.01]$. Contrasts indicated that the change from conditioning to test was greater for the $\mathrm{ML} / \mathrm{E}$ and $\mathrm{MT} / \mathrm{E}$ groups than for their $\mathrm{ML} / \mathrm{N}$ and $\mathrm{MT} / \mathrm{N}$ controls $[F(1,35)$ $=10.78, p<.005]$; however, it was not different for the pairs of ML and MT groups, and was not affected by the interaction of the extinction and stimulus factors. Importantly, though, the change in the difference-score measure was significantly greater for the MT/E group than for the ST/E group $[F(1,35)=4.95, p<.05]$. Consistent with those effects, separate group assessments of the change from conditioning to test indicated that the $M L / E$ and MT/E groups each had a significantly larger difference score during test $[F \mathrm{~s}(1,35)>4.80, p \mathrm{~s}<.05]$. In contrast, the $M L / N, M T / N$, and ST/E groups showed absolutely no change from conditioning to test $(F \mathrm{~s}<1)$.

\section{Retardation Testing}

Figure 3 shows the results of the retardation test, when $B$ itself was reinforced on a $50 \%$ basis, and during the pretest (PRE) day, when B was presented alone. As indicated, there were no reliable differences among the groups during the pretest, other than a stimulus effect showing greater suppression for the ML than for the MT subjects $[F(1,35)=70.06, p<.001]$. That difference corresponds to the stimulus difference observed in the habituation and extinction phases of training.

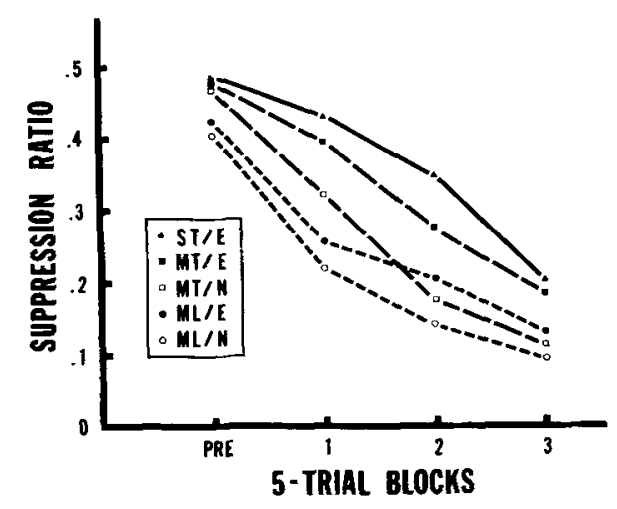

Figure 3. Mean suppression for each group to its respective $B$ inhibitor during both the pretest (PRE) and the retardation test. ML $=$ moderately developed light inhibitor; $M T=$ moderately developed tone inhibitor; $S T$ = strongly developed tone inhibitor; $E$ and $\mathbf{N}$ designate groups that received extinction training $(E)$ or not $(\mathbf{N})$.
During the retardation test, substantial suppression developed to $B$ for all groups, with the rate of acquisition being slower for the ST/E group and for the MT/E and ML/E groups as compared with their MT/N and ML/N controls. Thus, analysis of the data indicated a reliable group effect $[F(4,35)=6.34, p<.001]$. Contrasts showed that the MT/E and ML/E groups differed reliably from their $\mathrm{MT} / \mathrm{N}$ and $\mathrm{ML} / \mathrm{N}$ controls $[F(1,35)=5.54$, $p<.025]$ and that the pair of MT groups differed reliably from the pair of $\mathrm{ML}$ groups $[F(1,35)=6.36$, $p<.025]$. However, those extinction and stimulus effects did not interact. Furthermore, there was no reliable difference between MT/E and ST/E groups, despite the different extents to which their inhibitory CSs had been trained.

\section{DISCUSSION}

The present findings indicate that the inhibitory effect of a moderately developed $\mathrm{CS}$ - is enhanced when the $\mathrm{CS}$ is repeatedly presented by itself following $\mathrm{A}+/ \mathrm{AB}-$ training. This enhancement was evident in two ways: by the greater inhibitory effect of B for the $M T / E$ and $M L / E$ groups in summation and retardation testing relative to that for their MT/N and ML/N controls, and also by comparison with their own performance at the end of inhibitory conditioning, prior to the extinction phase. In contrast, the MT/N and ML/N controls showed no change in inhibitory performance from conditioning to test, indicating that the observed differences in test were not due, even in part, to a loss of inhibition for the controls.

The enhancement of $\mathrm{CI}$ for the $\mathrm{MT} / \mathrm{E}$ and $\mathrm{ML} / \mathrm{E}$ groups cannot be ascribed to a loss of unconditioned suppression to $B$ as a result of the $B$-alone trials given in the extinction phase because (1) unconditioned suppression to the intended tone inhibitor had completely dissipated by the end of habituation training, and (2) even if it were possible that subsequent conditioning had dishabituated unconditioned suppression to the tone, the dissipation of that suppression in the extinction phase would have occurred for both the MT/E and ST/E groups. However, the ST/E group showed absolutely no gain in inhibition from conditioning to test, despite the fact that it had not reached an asymptote of inhibitory performance (see Figure 1, left panel). These arguments are not weakened by the fact that there was some residual suppression to the intended light inhibitor at the end of habituation training. If a dissipation of unconditioned suppression to B were responsible for the enhancement effect, that factor would have operated primarily, if not solely, for the ML/E group and yielded an interaction between extinction training and the type of stimulus (light or tone) employed as the CS-. However, there was no reliable interaction of the extinction and stimulus factors in test.

Likewise, the enhancement of CI cannot be ascribed to ancillary effects, such as a change in the associative value of the training context. By that account, extinction training could have reduced the excitation that could pos- 
sibly have accrued to the contextual cues by way of their association with the shock and/or the A excitor in the conditioning phase. Hence, there would have been less excitation for $\mathbf{B}$ to overcome when those weakened excitatory cues of the context occurred in conjunction with the A excitor during the summation test or on their own during the retardation test. That argument, however, suffers for two reasons: First, it calls for an enhancement of CI for all of the extinguished groups, including the ST/E group; but, as noted, that group showed no gain in inhibitory performance from conditioning to test. Second, the argument implies that all of the extinguished groups would show less suppression to the A excitor when that CS was presented in conjunction with the weakened excitatory cues of the context, following the extinction phase. However, there were no differences in suppression to $A$ between extinguished and nonextinguished groups, either in the summation test or on the $A$ trials immediately preceding that test.

$A$ basis for the enhancement of $C I$ to $B$ is apparent in the behavior that the $\mathrm{MT} / \mathrm{E}$ and $\mathrm{ML} / \mathrm{E}$ groups exhibited in the extinction phase (see Figure 1, right panel). At the start of that phase, those groups showed greater suppression to $B$, not only in comparison with the ST/E group, but also in comparison with their own levels of unconditioned suppression to $B$ at the end of habituation training. The increase in suppression suggests that, in addition to B's moderately developed inhibitory property, that is, as a signal for nonreinforcement, $B$ acquired some second-order excitation from its association with $\mathrm{A}$ in the A + /AB - phase (e.g., Holland \& Rescorla, 1975; Rescorla, 1973). To use more current terminology, B's moderate inhibitory effect was insufficient to overcome the excitatory representation of A that B evoked (cf. Cunningham, 1981; Rescorla, 1981, 1982; Williams et al., 1986). Given that relationship, nonreinforced presentations of $B$ in the extinction phase would have two effects: (1) They would strengthen B's property as a signal for nonreinforcement in a manner analogous to B's development as a CS - during Pavlovian training, that is, when $B$ was nonreinforced in the presence of the excitation produced by the A stimulus itself (cf. Rescorla \& Wagner, 1972); and (2) they would disassociate B from A, thereby reducing the excitatory representation of $A$ that $B$ could evoke (Rescorla, 1982; Williams et al., 1986). Both of those effects would attenuate suppression to $B$ over the course of the extinction phase, as was observed for MT/E and the $\mathrm{ML} / \mathrm{E}$ groups, but only the former would enable $B$ to yield a stronger inhibitory effect when subsequently summated with $\mathrm{A}$. That follows because B's disassociation from $A$ would not reduce the excitation produced by the A stimulus itself. In support of that analysis, as noted, there were no differences in suppression to $\mathrm{A}$ between extinguished and nonextinguished groups in the summation test or on the $A$ trials immediately preceding that test.

The above interpretation also accommodates the effects of extinction training for a strongly developed CS - and, in the process, offers a counterintuitive prediction on the transfer of an inhibitor to a new excitor. When B's inhibitory property is sufficiently developed to substantially overcome the excitatory effect of $A$, as it was for the ST/E group (see Figure 1, left panel), that strong inhibition will completely offset the relatively weak excitatory representation of $A$ that B evokes, and therefore B will not exhibit a net excitatory effect when presented alone (see Figure 1, right panel). Furthermore, in the absence of such an effect, nonreinforced presentations of $B$ will fail to enhance B's inhibitory property and therefore, as observed for the ST/E group, there will be no gain in B's inhibitory effect when $B$ is subsequently summated with A. However, because extinction training still allows B's disassociation from A, B's inhibitory effect will seemingly be augmented if $B$ is tested with a new excitor, C (Rescorla, 1982; Williams et al., 1986). This enhancement, though, is due solely to the loss in the excitatory representation of $A$ that would otherwise summate with $C$ if $B$ were not given extinction training and disassociated from A. Accordingly, when $A$ is extinguished prior to $B$, the B extinction trials have no added effect on B's summation with C (Rescorla, 1982). With one exception, the same transfer effects should occur for a moderately developed $\mathrm{CS}-$. In this case, nonreinforced presentations of $\mathbf{B}$ will not only disassociate $\mathbf{B}$ from $\mathbf{A}$, but will also enhance B's inhibitory property, for the reasons earlier considered. Hence, the net gain in inhibition when $B$ is summated with $\mathrm{C}$ should be greater for a moderately developed CS - than for a strongly developed CS - . To our knowledge, this prediction has not been assessed.

The above findings on the transfer of a strongly developed CS - to a new excitor are important because they refute an alternative account of the observed enhancement effect. One could argue that the second-order excitation accruing to $B$ as a result of its pairing with $A$ in $A+/ A B-$ training is a transient effect that operates for a moderately, but not for a strongly, developed CS - . Consequently, the extinction of that excitation in $\mathrm{B}$-alone training would enhance B's inhibitory effect for the MT/E and ML/E groups but not for the ST/E group. Although attractive in its parsimony, that argument runs aground because it fails to account for (1) the enhanced transfer of inhibition that results from the extinction of a strongly developed CS - , and (2) the fact that such enhancement stems from a loss of the excitation produced by a representation of A. In evidence of those effects, B's inhibitory effect on a new excitor, $C$, is enhanced when either $A$ or $\mathrm{B}$ is extingished following $\mathrm{A}+/ \mathrm{AB}-$ training (Rescorla, 1982; Williams et al., 1986), although, as noted, the $B$ extinction trials have no added effect when $A$ is extinguished prior to B (Rescorla, 1982). These findings establish that even a strongly developed CS - has a secondorder excitatory association that is mediated by A's representation, and that the loss of that representation can enhance B's inhibitory effect with $C$, but not with $A$, which follows because the excitatory effect of A's representation can summate with the excitation produced by a new stimulus, $C$, but not with that produced by $A$ itself. 


\section{REFERENCES}

Cunningham, C. L. (1981). Association between the elements of a bivalent stimulus. Journal of Experimental Psychology: Animal Behavior Processes, 7, 425-436.

DEVITO, P. L., \& FowLER, H. (1986). Effects of contingency violations on the extinction of a conditioned fear inhibitor and a conditioned fear excitor. Journal of Experimental Psychology: Animal Behavior Processes, 12, 99-115.

Henderson, R. W. (1978). Forgetting of conditioned fear inhibition. Learning \& Motivation, 9, 16-30.

HollaND, P. C. (1985). The nature of conditioned inhibition in serial and simultaneous feature negative discriminations. In R. R. Miller \& N. E. Spear (Eds.), Information processing in animals: Conditioned inhibition (pp. 267-297). Hillsdale, NJ: Erlbaum.

Holland, P. C., \&escorla, R. A. (1975). Second-order conditioning with food unconditioned stimulus. Joumal of Comparative \& Physiological Psychology, 88, 459-467.

Kleiman, M. C., \& Fowler, H. (1984). Variations in explicitly unpaired training are differentially effective in producing conditioned inhibition. Learning \& Motivation, 15, 127-155.

LYSLE, D. T. (1983). Nonassociative extinction of a conditioned inhibitor. Unpublished master's thesis, University of Pittsburgh.

LYSLE, D. T., \& FowLER, H. (1985). Inhibition as a "slave" process: Deactivation of conditioned inhibition through extinction of conditioned excitation. Journal of Experimental Psychology: Animal Behavior Processes, 11, 71-94.

OWREN, M. J., \& KAPLAN, P. S. (1981, April). On the failure to extinguish conditioned inhibition: A test of a reinstatement hypothesis. Paper presented at the meeting of the Midwestern Psychological Association, Detroit, MI.

Pearce, J. M., Nicholas, D. J., \& Dickinson, A. (1982). Loss of associability by a conditioned inhibitor. Quarterly Journal of Experimental Psychology, 33B, 149-162.
ResCoRla, R. A. (1973). Second-order conditioning: Implications for theories of learning. In F. J. McGuigan \& D. B. Lumsden (Eds.), Contemporary approaches to conditioning and learning (pp. 127-150). Washington, DC: V. H. Winston.

Rescorla, R. A. (1981). Within-signal learning in autoshaping. Animal Learning \& Behavior, 9, 245-252.

Rescorla, R. A. (1982). Some consequences of associations between the excitor and inhibitor in a conditioned inhibition paradigm. Jour nal of Experimental Psychology: Animal Behavior Processes, 8, 288-298.

Rescorla, R. A., \& Wagner, A. R. (1972). A theory of Pavlovian conditioning: Variations in the effectiveness of reinforcement and nonreinforcement. In A. H. Black \& W. H. Prokasy (Eds.), Classical conditioning II: Current research and theory (pp. 64-99). New York: Appleton-Century-Crofts.

Thomas, D. A. (1979). Retention of conditioned inhibition in a bar press suppression paradigm. Learning \& Motivation, 10, 161-177.

Wagner, A. R., \& Rescorla, R. A. (1972). Inhibition in Pavlovian conditioning: Application of a theory. In R. A. Boakes \& M. S. Halliday (Eds.), Inhibition and learning (pp. 301-336). New York: Academic Press.

Williams, D. A., Travis, G. M., \& Overmier, J. B. (1986). Withincompound associations modulate the relative effectiveness of differential and Pavlovian conditioned inhibition procedures. Journal of Experimental Psychology: Animal Behavior Processes, 12, 351-362.

Witcher, E. S., \& AYres, J. J. B. (1984). A test of two methods for extinguishing Pavlovian conditioned inhibition. Animal Learning \& Behavior, 12, 149-156.

Zimmer-HART, C. L., \& Rescorla, R. A. (1974). Extinction of Pavlovian conditioned inhibition. Journal of Comparative \& Physological Psychology, 86, 837-845.

(Manuscript received January 29, 1987; revision accepted for publication July 30,1987 .) 